\title{
Moments of the truncated multiplicity distributions
}

\author{
I.M. Dremin, V.A. Nechitailo \\ Lebedev Physical Institute, Moscow
}

\begin{abstract}
In experiment, the multiplicity distributions of inelastic processes are truncated due to finite energy, insufficient statistics or special choice of events. It is shown that the moments of such truncated multiplicity distributions possess some typical features.In particular, the oscillations of cumulant moments at high ranks and their negative values at the second rank can be considered as ones most indicative on specifics of these distributions. They allow to distinguish between distributions of different type.
\end{abstract}

\section{Introduction}

Studies of multiplicity distributions of high-energy inelastic processes have produced many important and sometimes unexpected results (for the reviews, see, e.g., [1, 2, 3]). The completely new region of very high multiplicities will be opened with the advent of RHIC, LHC and TESLA accelerators.

Theoretical approaches to multiplicity distributions in high-energy processes have usually to deal with analytic expressions at (pre)asymptotic energies which only approximately account for the energy-momentum conservation laws or with purely phenomenological expressions of the probability theory. The multiplicity range extends in this case from zero to infinity.

In experiment, however, one has to consider distributions truncated at some multiplicity values in one or another way. These cuts could appear due to energy limitations, low statistics of experimental data or because of special conditions of an experiment. Energy limitations always impose the upper cotoff on the tail of the multiplicity distributions. Low statistics of data can truncate these distributions from both ends if it is insufficient to detect rare events with very low and/or very high multiplicity. Similar truncations

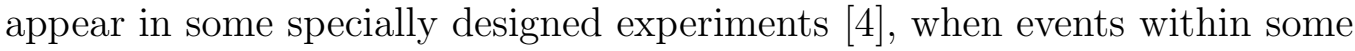
definite range of multiplicities have been chosen.

It would be desirable even in these cases to compare the distributions within those limited regions with underlying theoretical distributions. The 
straightforward fits are sometimes not accurate enough to distinguish between various possibilities because the probability values vary by many orders of the magnitude. More rigorous approach is to compare different moments of the truncated distributions. Among them, the cumulant moments $K_{q}$ seem to be most sensitive to slight variations (and, especially, cuts and shoulders) of the distributions. They often reveal such tiny details of the distributions which otherwise are hard to notice.

In particular, QCD predicts quite peculiar behaviour of cumulant moments as functions of their rank $q$. According to solutions of the equations for the generating functions of the multiplicity distributions in the asymptotic energy region, the ratio of cumulant moments $K_{q}$ to factorial moments $F_{q}$ usually denoted as $H_{q}=K_{q} / F_{q}$ behaves as $q^{-2}$ and at preasymptotic energy values reveals the minimum [5] at $q \approx 5$ with subsequent oscillations at higher ranks [6, 77. Such a behaviour has been found in experiment at presently available energies [8, 9]. The solutions of the corresponding equations for the fixed coupling QCD also indicate on similar oscillations [10]. At asymptotics, the oscillations should disappear and $H_{q}$ becomes a smoothly decreasing and positively definite function of $q$, as mentioned above.

Neither of the distributions of the probability theory possesses these features. Among them, the negative binomial distribution (NBD) happens to be one of the most successful ones in the description of global features of the multiplicity distributions [11]. Let us remind that the negative binomial distribution is defined as

$$
P_{n}=\frac{\Gamma(n+k)}{\Gamma(n+1) \Gamma(k)} a^{n}(1+a)^{-n-k},
$$

where $a=\langle n\rangle / k,\langle n\rangle$ is the mean multiplicity, $k$ is an adjustable parameter, and the normalization condition reads

$$
\sum_{n=0}^{\infty} P_{n}=1
$$

Its generating function is

$$
G(z)=\sum_{n=0}^{\infty} P_{n}(1+z)^{n}=\left(1-\frac{z\langle n\rangle}{k}\right)^{-k} .
$$


The integer rank factorial and cumulant moments, and their ratio are

$$
\begin{gathered}
F_{q}=\left.\frac{1}{\langle n\rangle^{q}} \frac{d^{q} G(z)}{d z^{q}}\right|_{z=0}=\frac{\Gamma(q+k)}{\Gamma(k) k^{q}} \\
K_{q}=\left.\frac{1}{\langle n\rangle^{q}} \frac{d^{q} \ln G(z)}{d z^{q}}\right|_{z=0}=\frac{\Gamma(q)}{k^{q-1}} \\
H_{q}=\frac{\Gamma(q) \Gamma(k+1)}{\Gamma(k+q)} .
\end{gathered}
$$

$H_{q}$-moments at the parameter $k=2$ behave as $2 / q(q+1)$, i.e. with the powerlaw decrease reminding at large $q$ that of QCD, however, with a different weight factor. Therefore, at first sight, it could be considered as a reasonably good analytic model for asymptotic behaviour of multiplicity distributions. It has been proclaimed [12, 13, 14, 15] that the superposition of two NBDs with different parameters and their cutoff at high multiplicities can give rise to oscillations of $H_{q}$ and better fits of experimental data at preasymptotic energies. Nevertheless, the fits have not been perfect enough.

Let us compare first the asymptotic QCD predictions with NBD fits at different values of the adjustable parameter $k$. The values of $D_{q}=q^{2} H_{q}$ are plotted in Fig. 1 as functions of $q$ for the asymptotic QCD (where they are identically equal to 1 ) and for NBD at different $k$. At $k=2$, they exceed 1 tending to 2 at large $q$. At larger values of $k$, all $D_{q}$ are less than 1 except $D_{2}=1$ at $k=3$. Surely, the identity $D_{1} \equiv 1$ is valid for any $k$ due to the normalization condition.

To get asymptotic QCD results with all $D_{q} \equiv 1$ from the expressions similar to NBD, one would need to modify NBD in such a way that the parameter $k$ becomes a function of $n$. Thus some effective values of $k$ should be used to get QCD moments $D_{q}=1$ at various $q$. These effective values are shown in Fig. 1 at the corresponding QCD points. They are obtained as the solutions of the equation

$$
\prod_{n=1}^{q-1}\left(1+\frac{k(q)}{n}\right)=q^{2},
$$

which follows from Eq. (6) for $H_{q}=q^{-2}$. They show that $k$ somewhat decreases from 3 to some values exceeding 2 with increase of $q$. This reflects 
the well known fact that the tails of distributions are underestimated in NBDfits [14 compared to experimental data in the preasymptotic region. Also, the amplitude of oscillations and their periodicity are not well reproduced by a single truncated NBD [14], and one has to use the sum of at least two NBDs to get a better fit. However, rather large values of $k$ were obtained in these fits. It implies, in fact, that the fit is done with the help of two distributions very close to Poissonian shapes because the Poisson distribution is obtained from NBD in the limit $k \rightarrow \infty$. Therefore, the tails are suppressed very strongly.

Here, we will not try to fit experimental data focusing our efforts on qualitative changes of moments when NBD is truncated, especially, as applied to studies of very high multiplicities.

In QCD considerations based on the equations for the generating functions for quark and gluon jets, the preasymptotic (next-to leading order etc) corrections give rise to oscillations of $H_{q}$. Even though they are of the higher order in the coupling strength, they appear mainly due to account of energy conservation in the vertices of Feynman diagrams but not due to considering the higher order diagrams which are summed in the modified perturbation theory series (see [3]). In the phenomenological approach, this would effectively correspond to the cutoff of the multiplicity distribution at some large multiplicity. Therefore, we intend here to study how strongly such a cutoff influences the NBD-moments, whether it produces oscillations of the cumulant moments, how strong they are, and, as a more general case, consider the moments of NBD truncated both at low and high multiplicities. This would help answer the question if the shape of the distribution in the limited region can be accurately restored from the behaviour of its moments. It could become especially helpful if only events with very high multiplicities are considered in a given experiment because of the above mentioned underestimation of tails in the NBD-fits.

\section{Truncated NBD and its moments}

In real situations, the multiplicity distribution is sometimes measured in some interval of multiplicities and one can try to fit by NBD the data available only in the restricted multiplicity range. Therefore, we shall consider the negative binomial distribution within the interval of multiplicities $m \leq n \leq N$ called 
$P_{n}^{(c)}$ and normalized to 1 so that

$$
\sum_{n=m}^{N} P_{n}^{(c)}=1
$$

Moreover, due to above reasoning and to simplify formulas we consider here only the case of $k=2$. The generalization to arbitrary values of $k$ is straightforward.

The generating function of the truncated distribution $G_{c}(z)$ can be easily found as

$$
G_{c}(z)=\sum_{n=m}^{N} P_{n}^{(c)}(1+z)^{n}=G(z)(1+z)^{m} \frac{f(z)}{f(0)}
$$

where

$$
\begin{gathered}
f(z)=1+m(1-x)-[1+(N+1)(1-x)] x^{N-m+1}, \\
x=b(1+z), \quad b=\frac{a}{1+a} .
\end{gathered}
$$

Correspondingly,

$$
f(0) \equiv f(z=0) \equiv f(x=b) .
$$

Using the above formulas for the factorial moments, one gets the following formula for the moments of the truncated distribution expressed in terms of the NBD-moments (田):

$$
\begin{array}{r}
\langle n\rangle_{c}^{q} F_{q}^{(c)}=\sum_{r=0}^{q} \frac{\Gamma(q+1)}{\Gamma(r+1) \Gamma(q-r+1)}\langle n\rangle^{q-r} F_{q-r} . \\
\frac{\Gamma(m+2)}{\Gamma(m+2-r)}[m(1-b)+1-r]-\frac{\Gamma(N+3)}{\Gamma(N+3-r)} b^{N-m+1}[(N+1)(1-b)+1-r] \\
m(1-b)+1-b^{N-m+1}[(N+1)(1-b)+1]
\end{array}
$$

where $\langle n\rangle_{c}$ is the mean multiplicity of the truncated distribution. It is related to the mean multiplicity $\langle n\rangle$ of the original distribution as

$$
\langle n\rangle-\langle n\rangle_{c}=\frac{(1-b)\left[(N+1)(N+2) b^{N-m+1}-m(m+1)\right]}{1+m(1-b)+b^{N-m+1}[(N+1) b-N-2]} .
$$

Inserting formula (4) in (13), one gets

$$
\begin{gathered}
\langle n\rangle_{c}^{q} F_{q}^{(c)}=\sum_{r=0}^{q} \frac{(q-r+1) \Gamma(q+1)}{2^{q-r} \Gamma(r+1)}\langle n\rangle^{q-r} . \\
\frac{\Gamma(m+2)}{\Gamma(m+2-r)}[m(1-b)+1-r]-\frac{\Gamma(N+3)}{\Gamma(N+3-r)} b^{N-m+1}[(N+1)(1-b)+1-r] \\
m(1-b)+1-b^{N-m+1}[(N+1)(1-b)+1]
\end{gathered}
$$


For computing it is more convenient to use the formula (15) in the following form:

$$
\begin{aligned}
F_{q}^{(c)}= & \left(\frac{\langle n\rangle}{\langle n\rangle_{c}}\right)^{q} F_{q}\left\{1+\frac{1}{f(0)(q+1)} \sum_{r=1}^{q} \frac{a^{-r}}{r !}(q+1-r) \times\right. \\
& {\left[\left(\frac{m}{1+a}+1-r\right) \theta(m+1-r) \prod_{i=1}^{r}(m+2-i)\right.} \\
& \left.\left.-\left(\frac{N+1}{a+1}+1-r\right) b^{N-m+1} \prod_{i=1}^{r}(N+3-i)\right]\right\}
\end{aligned}
$$

These expressions can be used also for the distributions truncated at one side by setting $m=0$ or $N=\infty$.

The cumulant moments can be calculated after the factorial moments are known from Eq. (13) according to the identities

$$
F_{q}=\sum_{m=0}^{q-1} \frac{\Gamma(q)}{\Gamma(m+1) \Gamma(q-m)} K_{q-m} F_{m} .
$$

This formula is a simple relation between the derivatives of a function and of its logarithm (see Eqs (4) and (5)). Therefore it is valid for both original and truncated distributions.

For the Poisson distribution, the ratios $H_{q}$ are identically equal to zero, and are given by Eq. (6) for NBD while truncation induces new features. In Figures we show the behaviour of the ratios $H_{q}$ as functions of the rank $q$ for the truncated Poisson and negative binomial distributions.

At the beginning, we consider the abrupt cutoff only of the very high multiplicity tail, i.e., the case $m=0$ and $N>\langle n\rangle$. This mimics the energymomentum conservation limits. In Figs 2 and 3, it is shown that such a cutoff $N$ induces oscillations of $H_{q}$. The farther is the cutoff from the mean multiplicity, the weaker are oscillations. This quite expected result is known from long ago [12, 13]. It is demonstrated in Fig. 2 for $\langle n\rangle=10$ and different cutoffs at $N=30,40,50$. Another representation of the same result is seen in Fig. 3 where the constant cutoff $N=30$ has been chosen for different $\langle n\rangle$ equal to 5, 10 and 15 . The closer is the cutoff to $\langle n\rangle$, the stronger the low-rank moments are damped. For the faraway cutoff $N=50$, the period of oscillations increases. This increase is larger for lower mean multiplicity 
(see Fig. 4). At $N /\langle n\rangle=$ const, one observes the approximate scaling of $H_{q}$ as seen in Fig. 5.

\section{$3 \quad$ Very high multiplicities}

With the advent of RHIC, LHC and TESLA we are approaching the situation when average multiplicities become very high and the tails of multiplicity distributions reach the values which are extremely large. These events with extremely high multiplicities at the tail of the distribution can be of a special interest. The tails of particular channels die out usually very fast, and a single channel dominates at the very tail of the distribution. Mostly soft particles are created in there. Thus one hopes to get the direct access to very low- $x$ physics. QCD-interpretation in terms of BFKL-equation (or its generalization) can be attempted. Also, the hadronic densities are rather high in such events, and the thermodynamical approach can be applied [16].

However, these events are rather rare and the experimental statistics is quite poor until now. The Poisson distribution has the tail which decreases mainly like an inverse factorial. According to NBD (1), the tail is exponentially damped with the power-increasing preexponential factor. At the same time, QCD predicts even somewhat slower decrease as is seen in Fig. 1 from the behaviour of moments. This is important for future experiments in the very high multiplicity region.

To study these events within the truncated NBD with $k=2$ according to Eqs (15), (16), let us choose the multiplicity interval of the constant length $N-m=20$ and place it at various distances from the mean multiplicity $\langle n\rangle=10$ as in Fig. 2. The resulting $H_{q}$ are shown in Fig. 6. The most dramatic feature is the negative values of $\mathrm{H}_{2}$ and the subsequent change of sign of $H_{q}$ at each $q$ in the case when the lower cutoff $m$ is noticeably larger than $\langle n\rangle(m /\langle n\rangle \geq 2)$. This reminds of the behaviour of $H_{q}$ for the fixed multiplicity distribution and shows that the NBD-tail decreases quite fast so that the multiplicity $m$ dominates in the moments of these truncated distributions.

The same features are demonstrated in Figs 7 and 8 for different average multiplicities and different positions of the fixed window $N-m=20$. In Fig. 7 , the window is rather close to $\langle n\rangle$ or even contains it inside (for $\langle n\rangle=15$ ). Therefore $H_{2}<0$ only for $\langle n\rangle=5$. In Fig. 8 , it is very far from $\langle n\rangle$. Thus 
all $H_{2}$ are negative, the more the lower is $\langle n\rangle$. Again, the sign-changing characteristics remind those for the fixed multiplicity distribution.

Another possibility to study the tail of the distribution with the help of $H_{q}$-ratios is their variation with the varying length of the tail chosen. At the same mean multiplicity $\langle n\rangle=10$, we calculate moments for the intervals starting at $m=20$ and ending at $N=50,40,30,25$. The values of $H_{q}$ at rather low ranks $q=2,3,4,5$ are very sensitive to the interval length as shown in Fig. 9. The values of $H_{2}$ vary, e.g., by the order of magnitude.

\section{Conclusions}

In connection with some experiments planned, our main concern here was to learn if $H_{q}$-ratios can be used to judge about the behaviour of the tail of the multiplicity distribution. Using NBD as an example, we have shown that $H_{q}$ behave in a definite way depending on the size of the multiplicity interval chosen and on its location. Comparing the corresponding experimental results with NBD-predictions, one would be able to show whether the experimental distribution decrease slower (as predicted by QCD) or faster than NBD.

In particular, the negative values of $H_{2}$ noted above are of special interest because they show directly how strong is the decrease of the tail. NBDs at different $k$ values would predict different variations of $H_{2}$ with more negative $H_{2}$ for larger $k$. Also, the nature of oscillations of $H_{q}$-moments at larger values of $q$ reveals how steeply the tail drops down.

Let us stress that the choice of high multiplicities for such a conclusion could be better than the simpleminded fit of the whole distribution. As one hopes, in this case there is less transitions between different channels of the reaction (e.g., from jets with light quarks to heavy quarks), and the underlying low- $x$ dynamics can be revealed.

\section{Acknowledgements}

This work is supported by the RFBR grants N 00-02-16101 and 02-0216779.

\section{Figure captions.}

Fig. 1. $D_{q}=q^{2} H_{q}$ are shown for asymptotical QCD by circles $\left(D_{q}^{Q C D} \equiv\right.$ 
1) in comparison with NBD-predictions at different values of the parameter $k=2$ (diamonds), 3 (crosses), 10 (squares). The numbers near the QCD values show which values of $k$ one would need to use for NBD to fit $D_{q}=1$ at the corresponding $q$.

Fig. 2. $H_{q}$-moments for NBD with $\langle n\rangle=10$ truncated at $N=30$ (diamonds), 40 (crosses), 50 (squares) at $m=0$.

Fig. 3. $H_{q}$-moments for NBD with $\langle n\rangle=5$ (diamonds), 10 (crosses), 15 (squares) truncated at $N=30$ at $m=0$.

Fig. 4. $H_{q}$-moments for NBD with $\langle n\rangle=5$ (diamonds), 10 (crosses), 15 (squares) truncated at $N=50$ at $m=0$.

Fig. 5. Approximate scaling of $H_{q}$-moments for NBD with $N /\langle n\rangle=$ const.

Fig. 6. $H_{q}$-moments for NBD with $\langle n\rangle=10$ truncated at $m=10, N=30$ (diamonds), 20-40 (crosses), 30-50 (squares).

Fig. 7. $H_{q}$-moments for NBD with $\langle n\rangle=5$ (diamonds), 10 (crosses), 15 (squares) truncated at $m=10, N=30$.

Fig. 8. $H_{q}$-moments for NBD with $\langle n\rangle=5$ (diamonds), 10 (crosses), 15 (squares) truncated at $m=30, N=50$.

Fig. 9. $H_{q}$-moments for NBD with $\langle n\rangle=10$ truncated at $m=20, N=50$ (diamonds), 20-40 (crosses), 20-30 (squares), 20-25 (circles).

\section{References}

[1] I.M. Dremin, Phys.-Uspekhi 37, 715 (1994).

[2] E.A. DeWolf, I.M. Dremin and W. Kittel, Phys. Rep. 270, 1 (1996).

[3] I.M. Dremin and J.W. Gary, Phys. Rep. 349, 301 (2001).

[4] V.A. Nikitin, Talk at III International workshop on Very High Multiplicities, Dubna, June 2002.

[5] I.M. Dremin, Phys. Lett. B 313, 209 (1993).

[6] I.M. Dremin and V.A. Nechitailo, Mod. Phys. Lett. A 9, 1471 (1994); JETP Lett. 58, 881 (1993).

[7] S. Lupia, Phys. Lett. B 439, 150 (1998).

[8] I.M. Dremin, V. Arena, G. Boca et al, Phys. Lett. B 336, 119 (1994). 
[9] SLD Collaboration, K. Abe et al, Phys. Lett. B 371, 149 (1996).

[10] I.M. Dremin and R.C. Hwa, Phys. Rev. D 49, 5805 (1994); Phys. Lett. B 324, 477 (1994).

[11] A. Giovannini, Nuovo Cim. A 15, 543 (1973).

[12] R. Ugoccioni, A. Giovannini and S. Lupia, in M. M. Block, A.R. White (Eds.) Proc. XXIII Int. Symposium on Multiparticle Dynamics, Aspen, USA, 1993, WSPC, Singapore, 1994, p. 297.

[13] B.B. Levtchenko, in B.B. Levtchenko (Ed.), Proc. VIII Workshop on High Energy Physics, Zvenigorod, Russia, 1993, MSU, 1994, p. 68.

[14] A. Giovannini, S. Lupia and R. Ugoccioni, Phys. Lett. B 388, 639 (1996); 342, 387 (1995).

[15] R. Ugoccioni and A. Giovannini, Nucl. Phys. Proc. Suppl. 71, 201 (1999).

[16] J. Manjavidze and A. Sisakian, Phys. Rep. 346, 1 (2001). 


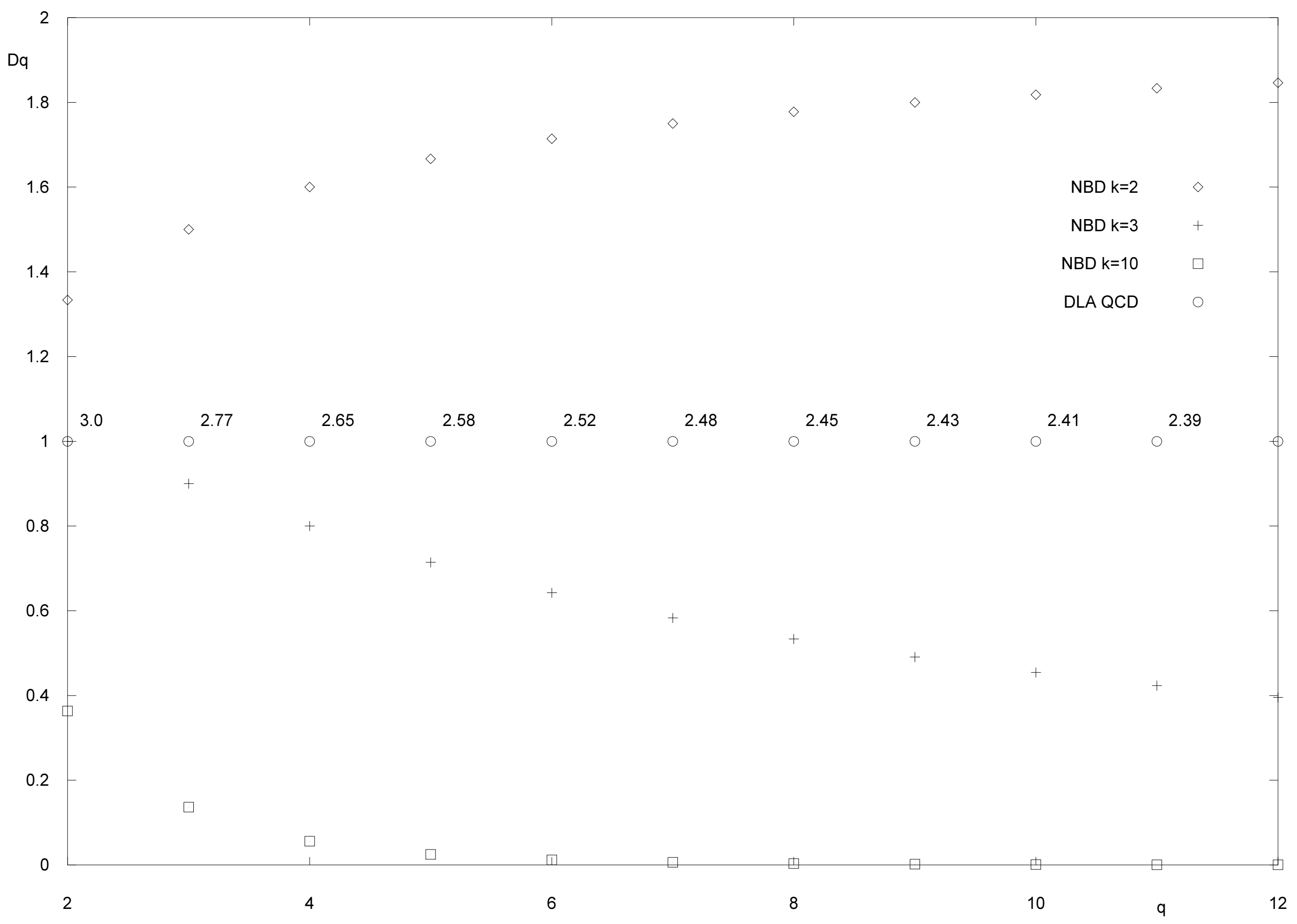




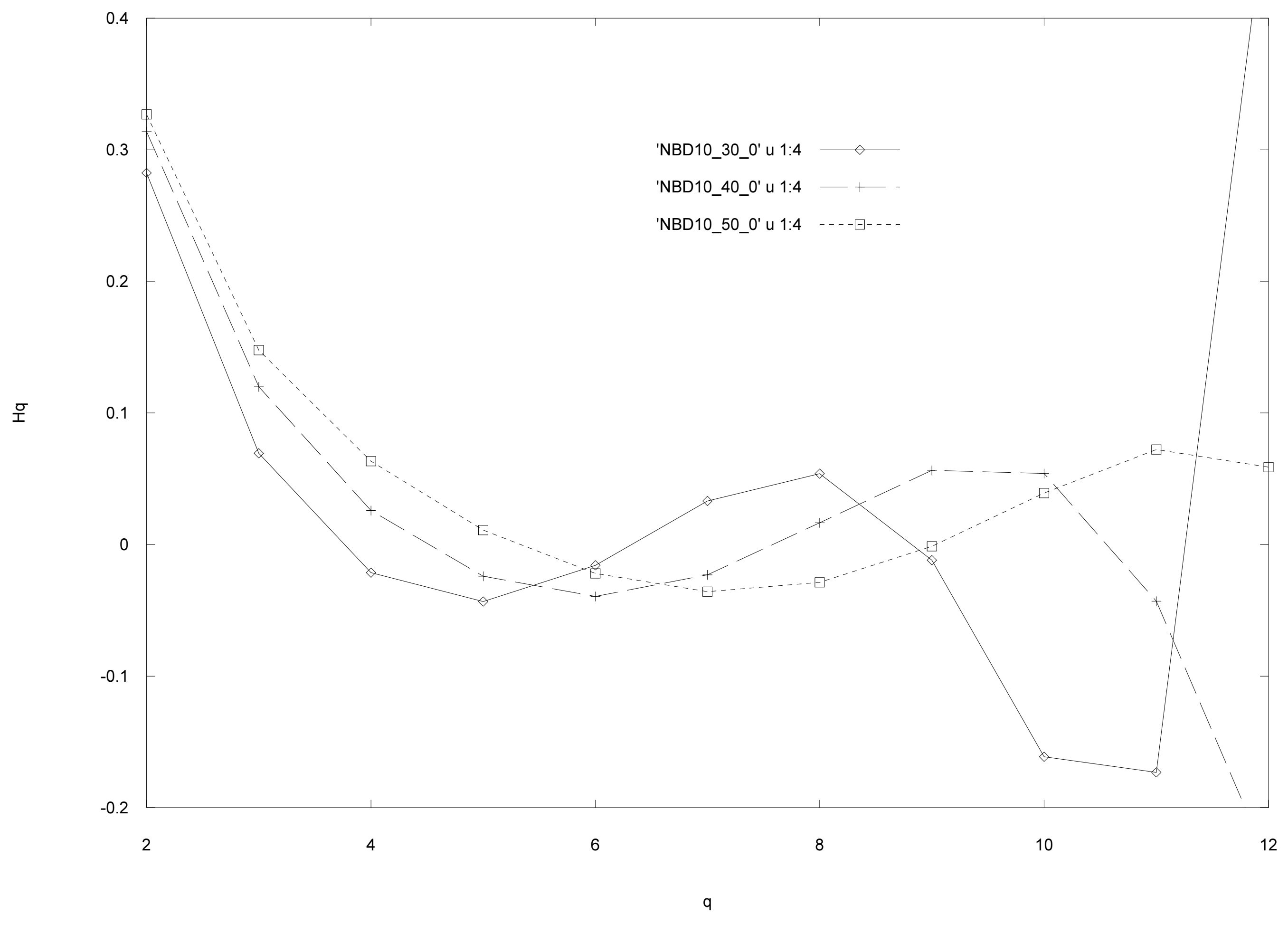




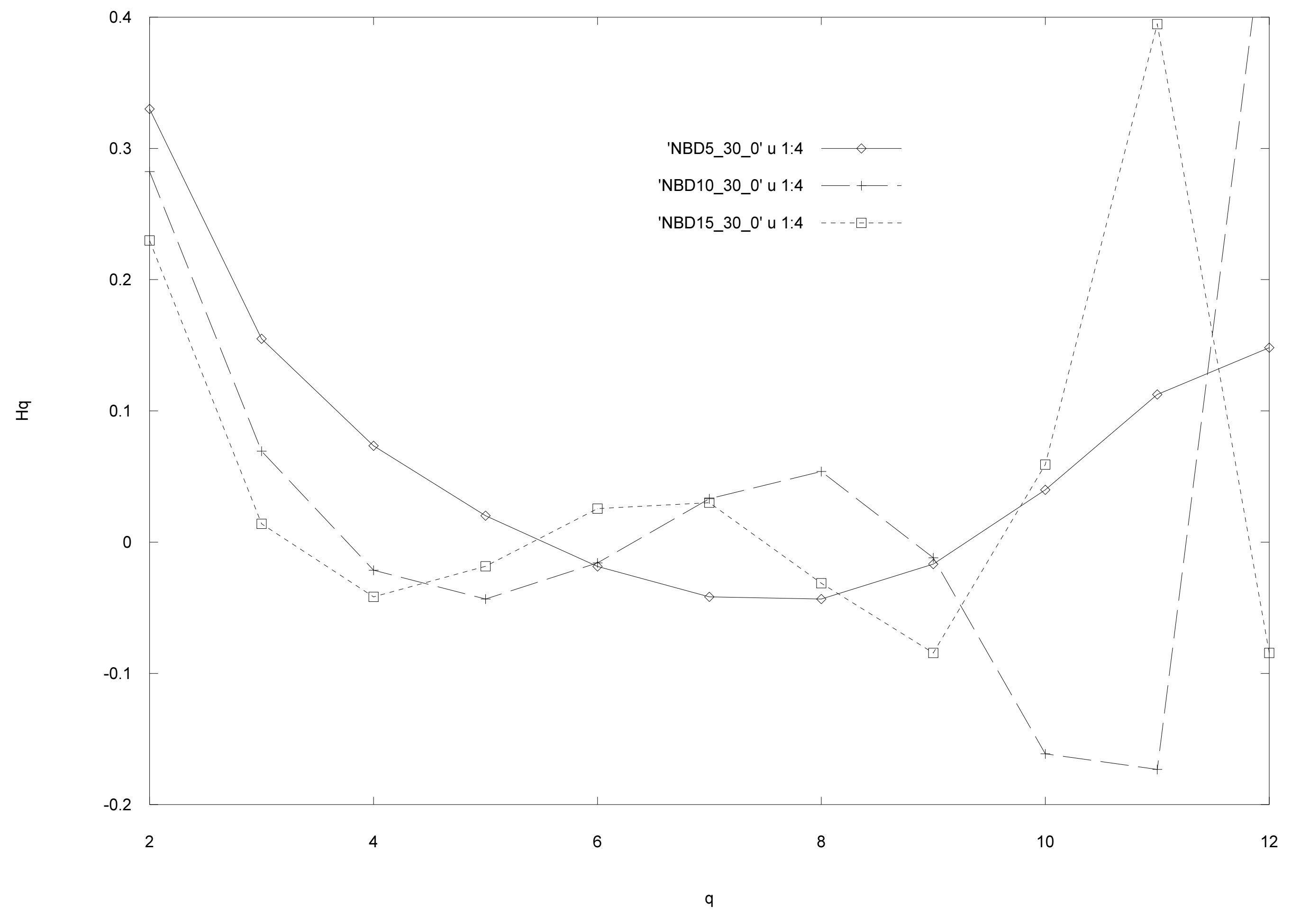




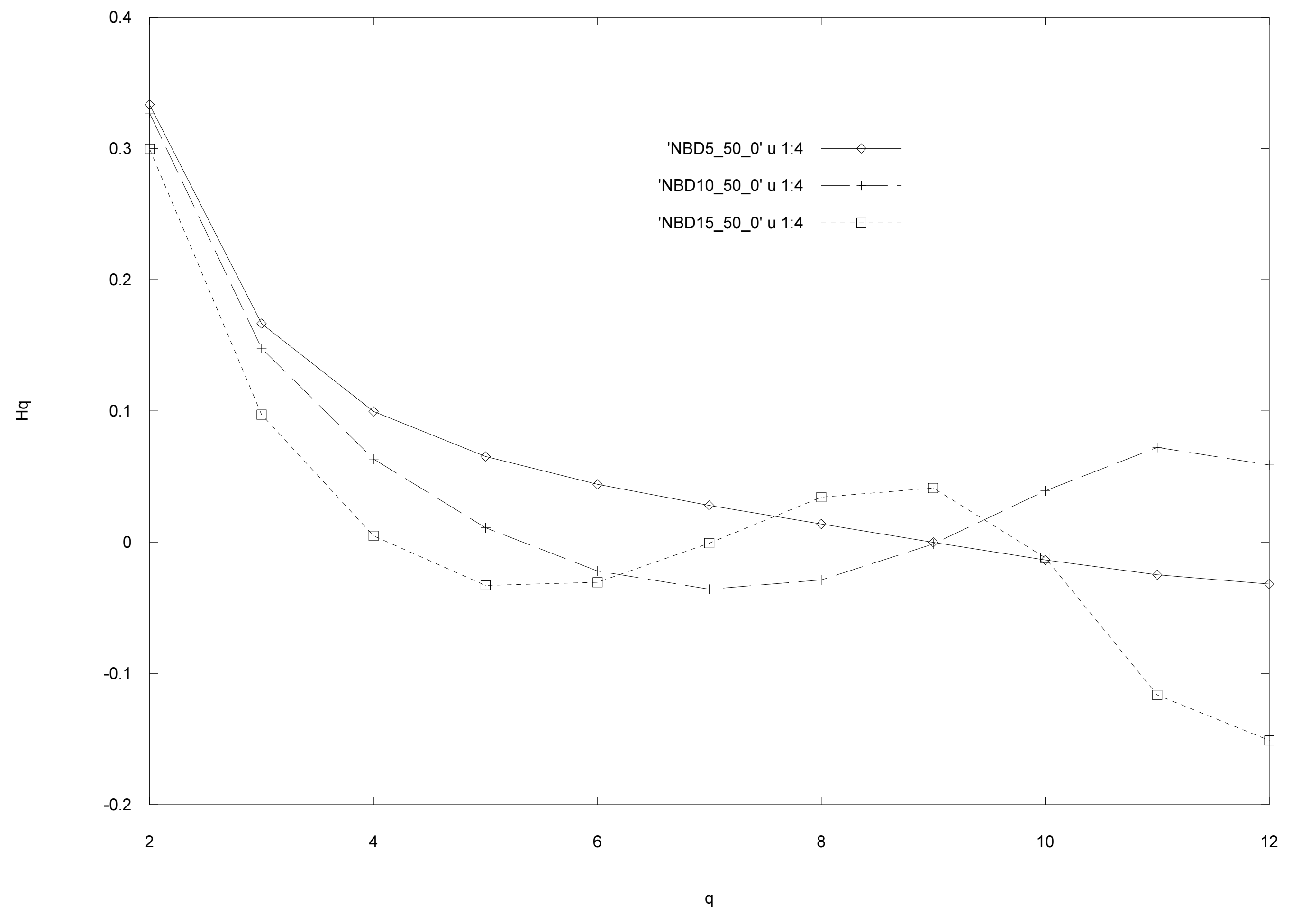




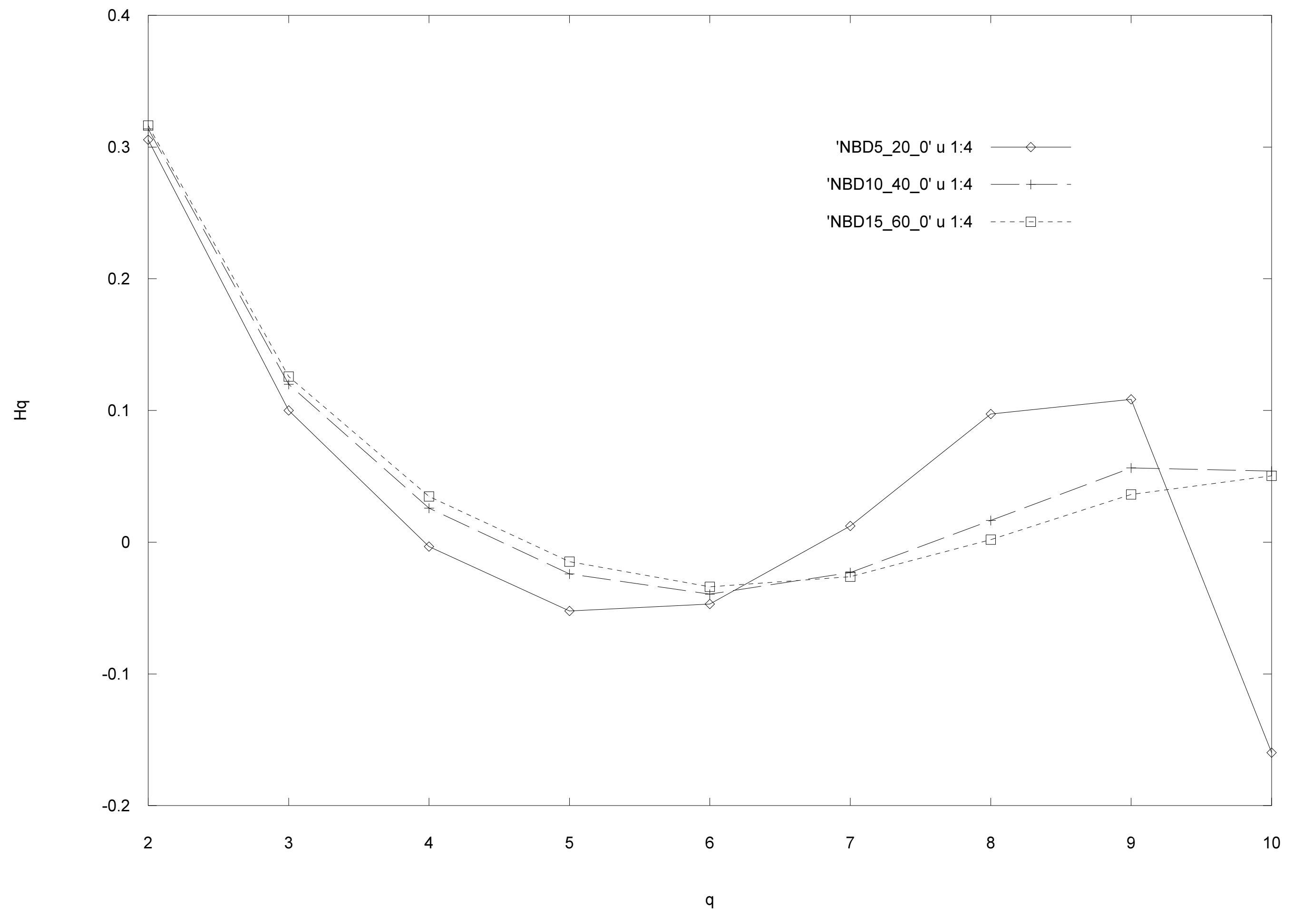




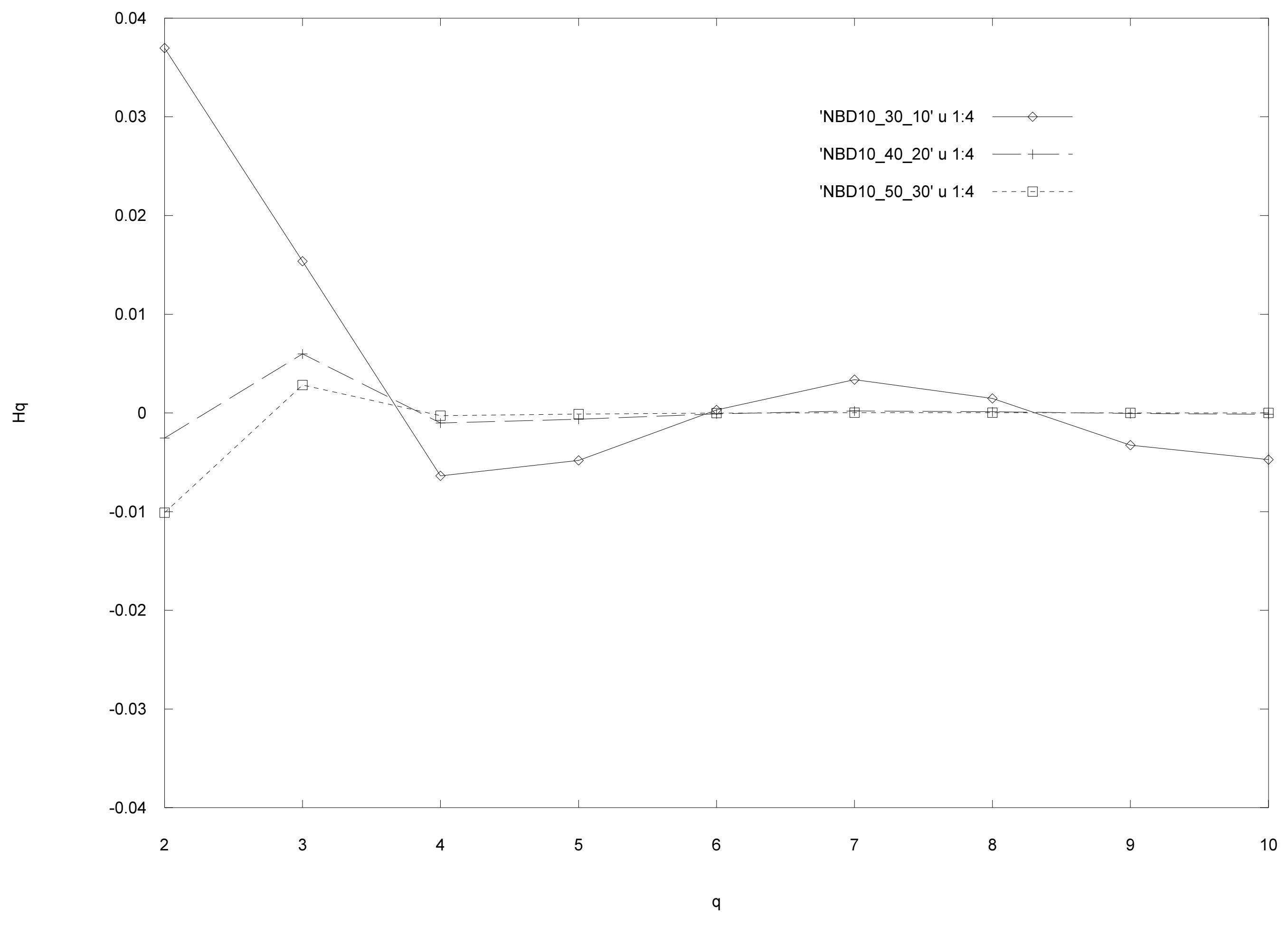




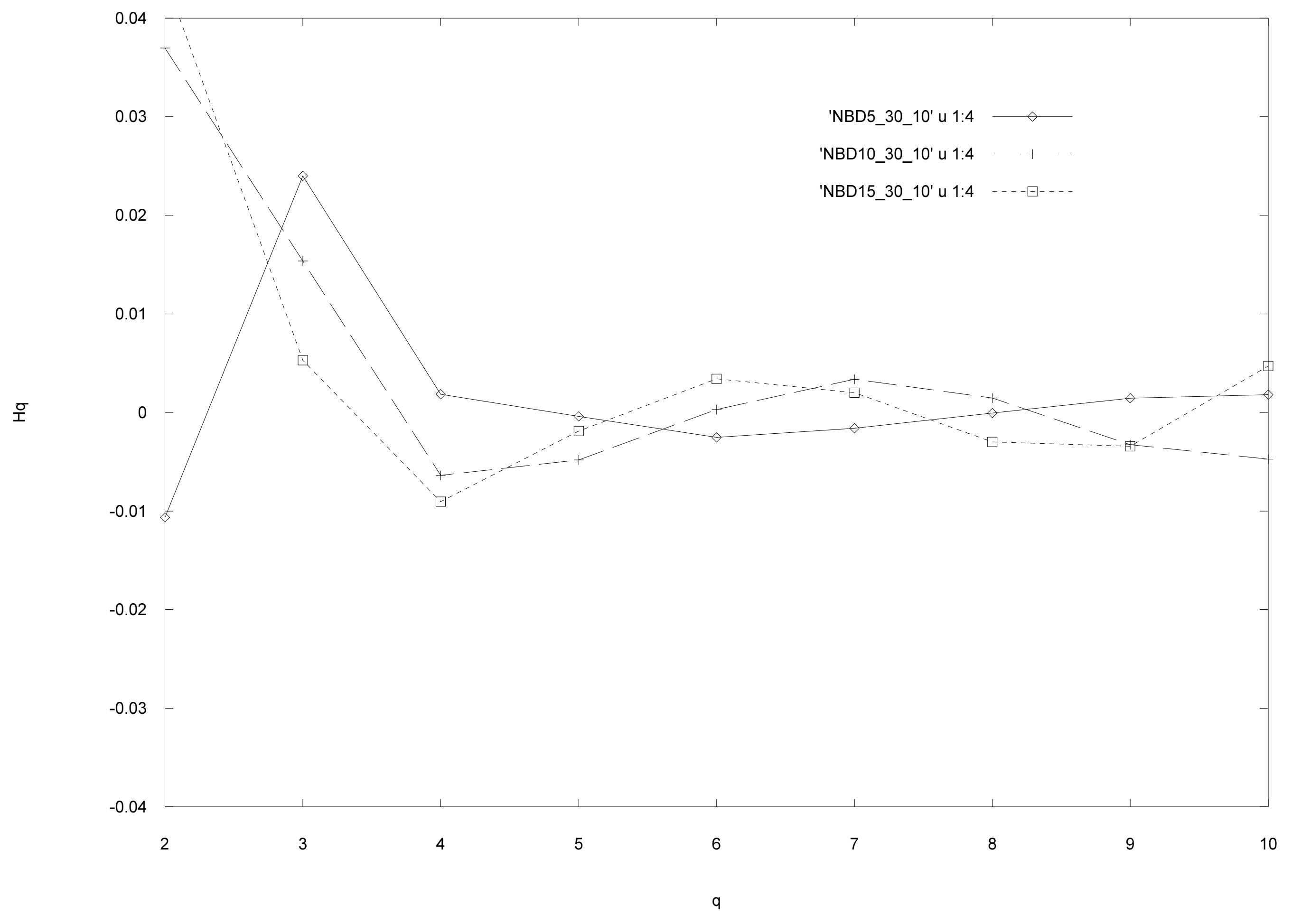




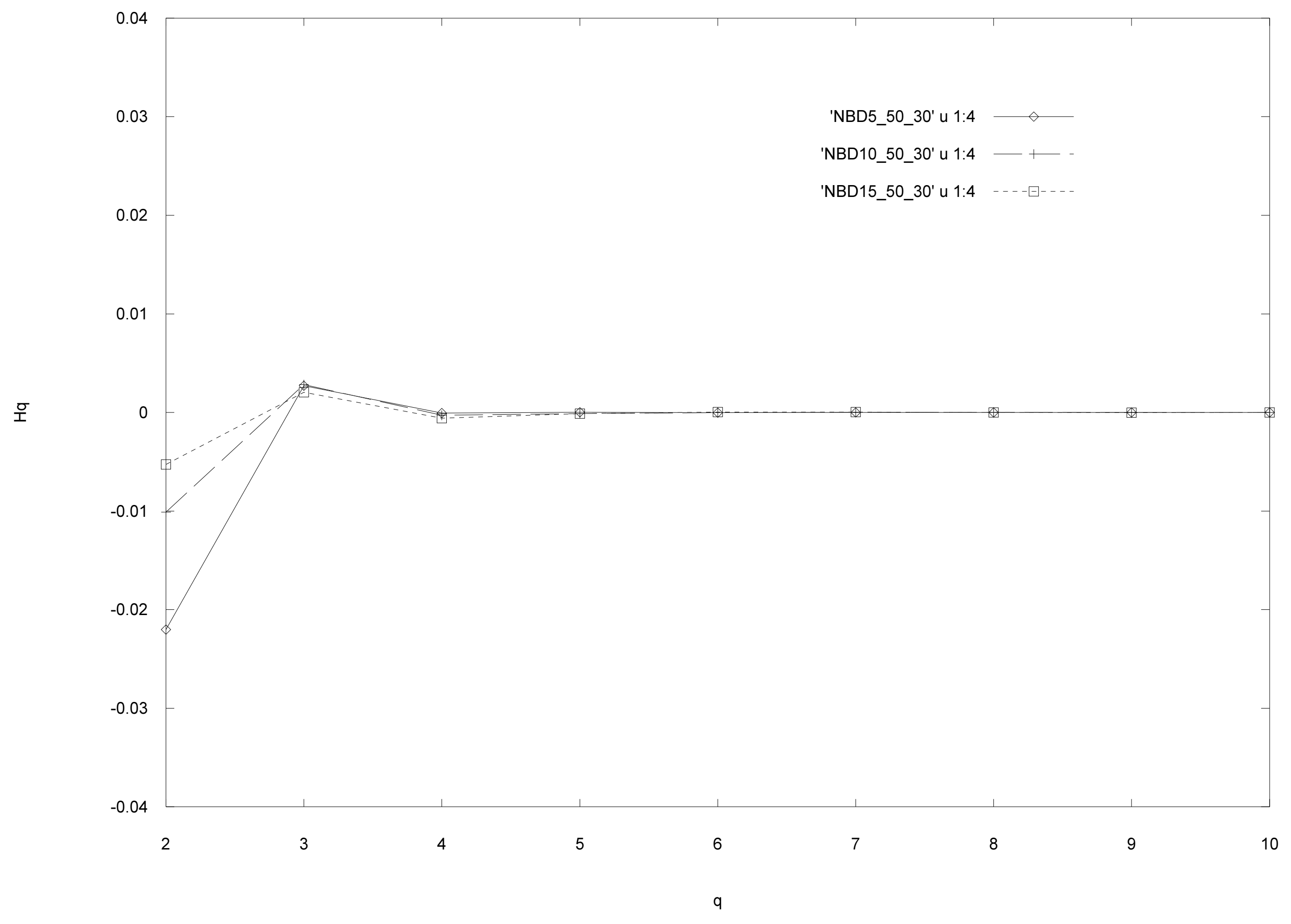




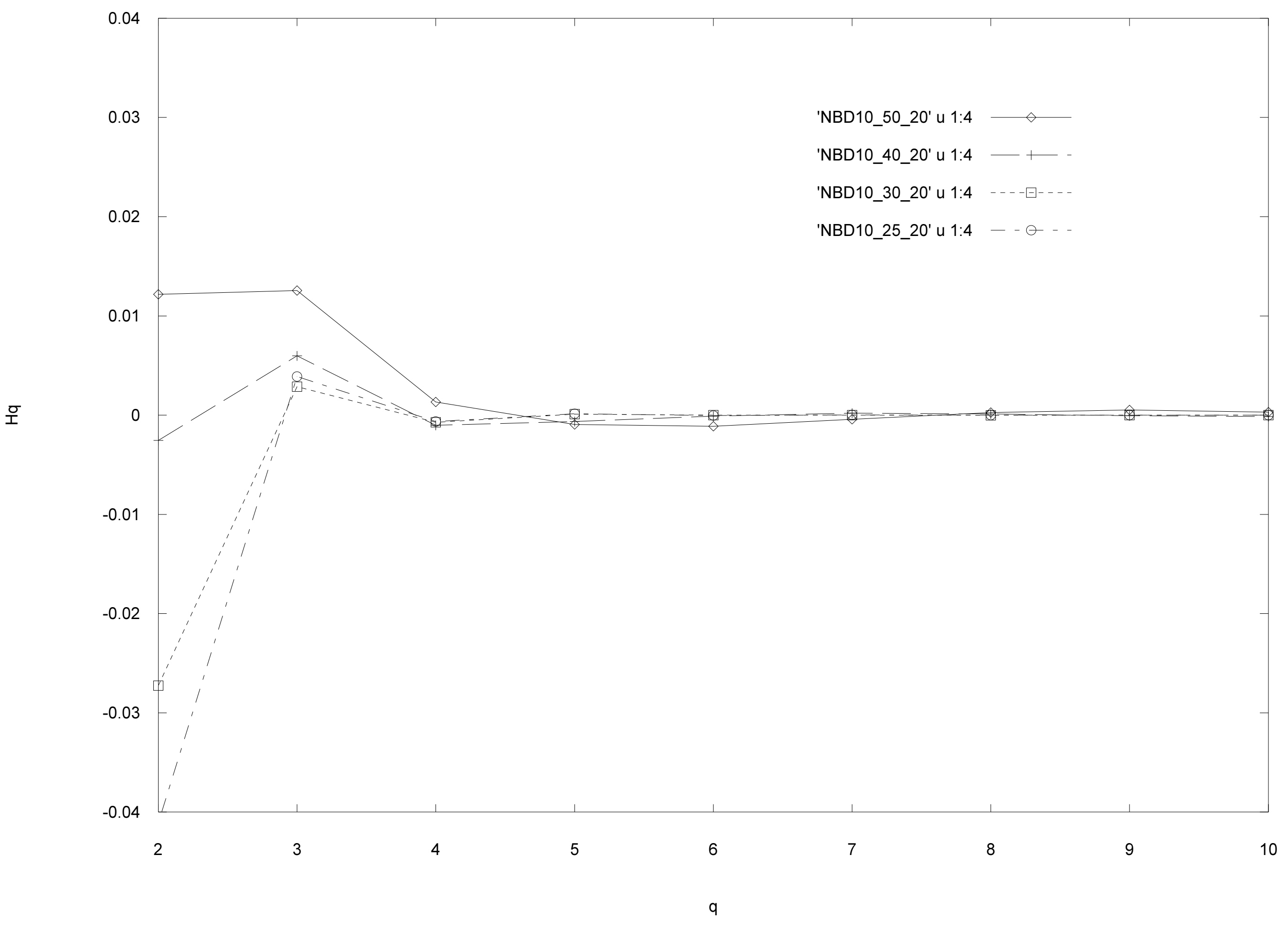

